\title{
High prevalence of dementia among community dwelling older adults in receipt of state funded home care packages: implications for health care planning
}

\author{
I. O'Brien ${ }^{1,2}$, K. Smuts ${ }^{1,2}$, C. W. Fan ${ }^{3}$, M. O'Sullivan ${ }^{4}$ and A. Warters ${ }^{5, *}$ \\ ${ }^{1}$ North Dublin Homecare Ltd., Dublin, Ireland \\ ${ }^{2}$ Community Healthcare Organisation, Ballymun Healthcare Facility, Dublin, Ireland \\ ${ }^{3}$ Department of Geriatric Medicine, Mater Misericordiae University Hospital, Dublin, Ireland \\ ${ }^{4}$ Clinical Medicine, Trinity Centre for Health Sciences, St. James's Hospital, Dublin, Ireland \\ ${ }^{5}$ Services for Older People, Health Service Executive, Community Healthcare Organisation, Ballymun Healthcare Facility, Dublin, Ireland
}

Objectives. The majority of people living with dementia in Ireland reside in their own homes, some supported by formal or informal home care. This audit aimed to estimate the prevalence of dementia and suspected cognitive impairment (CI) among older adults, 65+ years, in receipt of formal home care (domiciliary care) in a defined health service area in North Dublin. A secondary objective of the audit was to explore factors associated with dementia or CI in this cohort.

\begin{abstract}
Methods. A cross-sectional audit was conducted on all clients aged 65+ years actively receiving publicly funded home care packages (HCPs) during May 2016 in Healthcare Service Executive CHO9 Dublin North Central. A total of 935 urban community dwelling older adults were included in the study [mean age 83.7 (s.D. 7.4) years and 65\% female]. Basic socio-demographic and health data were extracted from common summary assessment reports. Service users were categorised as having (a) dementia if a diagnosis of dementia or cognitive decline which impacts on independent living, was documented by a health professional or (b) suspected CI where a validated cognitive screening tool was applied and the score was indicative of mild CI.
\end{abstract}

Results. Overall, the estimated prevalence of dementia and suspected CI was $37.1 \%$ and $8.7 \%$, respectively. Factors significantly associated with dementia and suspected CI were higher dependency and home care hours, communication difficulty and being non-self-caring $(p<0.001)$. Notably, half $(51.6 \%)$ of those with either dementia or suspected CI group lived alone.

Conclusions. Our findings suggest a high prevalence dementia among HCP users, highlighting a need and opportunity for dementia-specific approaches to support older people in their homes.

Received 20 June 2017; Revised 28 October 2017; Accepted 14 November 2017

Key words: Cognitive impairment, domiciliary care, home care, older adults.

\section{Introduction}

It is estimated that just over 50000 older adults aged 65 or over are living with dementia in Ireland with this number expected to more than triple by 2046 (Pierce et al. 2014). Of these, nearly two-thirds are thought to be living in the community (Pierce et al. 2014). The increase in prevalence of dementia represents a significant challenge for patients and families as well as existing health and social care systems. Home and communitybased supports have been recognised as critical to

* Address for correspondence: A. Warters, PhD, Community Healthcare Organisation 9, Ballymun Healthcare Facility, Dublin 9, Ireland.

(Email: wartersa@tcd.ie) enabling older people with dementia to remain living as active citizens in their own homes for as long as possible, as highlighted in the Irish National Dementia Strategy (Department of Health, 2014).

Given the progressive nature of dementia, community services need to be accessible and adaptable to the changing needs of the individual (Pierce et al. 2014). In Ireland, an estimated $8.2 \%$ of older people living in the community avail of state-funded home help (Murphy et al. 2015). Currently, the prevalence of dementia and cognitive impairment (CI) in this cohort remains undetermined, although it is anticipated that the level would be higher than in the general older population. The Dementia Strategy clearly states that 
'available resources should be deployed on the basis of need and as effectively as possible to provide services for all people with dementia' (Department of Health, 2014). The lack of Irish prevalence data amongst home care users makes it highly challenging to reconfigure resources and make decisions regarding future service planning, underscoring the need for the Irish Healthcare Service Executive (HSE) to generate such data, as outlined in previous literature (Cahill et al. 2012).

Moreover, people availing of home help services are in regular contact with community health and social care services, such as community nursing and home care. This represents an opportunity for deploying dementia-friendly services, supports and interventions for this population. Thus, establishing the prevalence of dementia in this cohort would be beneficial in implementing and co-ordinating such services.

The aim of this audit is to estimate the prevalence of dementia and suspected CI among older adults in receipt of state funded home care packages (HCPs). A HCP can be defined as a collection of community-based health and social care services which exceed the normal of 'generic' home help service provision offered by the HSE to individuals in the community. Generic home help services can be defined as 'a level of service that might reasonably be expected to be delivered to a client from the core budgets' or home help that amounts to $<5$ hours per week. Thus, HCPs can be seen as an enhanced level of community care for those who require additional care over and above the 'norm'. HCPs may include, but are not limited to, home care or home help, nursing, and therapies such as physiotherapy and occupational therapy. It is hypothesised that those living with dementia or suspected CI have higher rates of dependency as assessed by the Barthel Index and greater home care needs (hours per week) than those without dementia or suspected CI.

The audit took place in a specific HSE Local Health Office (LHO), namely Dublin North Central, in Community Healthcare Organisation (CHO) 9.

\section{Methods}

This audit was conducted on all clients aged 65 years and over actively receiving publicly funded HCPs during May 2016 in HSE CHO9 Dublin North Central. HCPs were defined as the provision of home care in excess of 5 hours per week. Information was extracted and anonymised from the common summary assessment reports (CSARs), a mandatory form completed by public health nurses as part of the HCP application and review process (Health Service Executive, 2009).

Basic socio-demographic data was available from the CSAR and documented for each HCP recipient. This included age, gender, marital status, living status, principal carer (the person who provides a significant amount of direct care, for example daily visits, provides meals; may be a paid carer) and communication ability (the ability to effectively communicate, retain and make sense of information related to the home care plan). Intensity of home care usage was measured based on weekly hours of care received. Dependence with activities of daily living (ADLs) was assessed using the modified Barthel Index with scores ranging from zero (complete dependence) to 20 (complete independence) (Collin et al. 1988). Need for prompting with medications was recorded as a binary variable.

Cognitive screening tests or information on dementia are not mandatory for HCP assessments in Ireland, but may be recorded on a case-by-case basis. For the purpose of this audit, service users were categorised as having (a) dementia if a diagnosis of dementia or cognitive decline with impact on independent living, was documented by a health professional (geriatrician, public health nurse, general practitioner, occupational therapist) or (b) suspected CI where a validated cognitive screening tool was applied and the documented score was indicative of mild CI. Service users with an absence of recorded evidence of a dementia diagnosis and those with a screening test score indicative of 'non- $\mathrm{CI}^{\prime}$ in the absence of other dementia evidence, were categorised as 'CI not suspected' (Fig. 1).

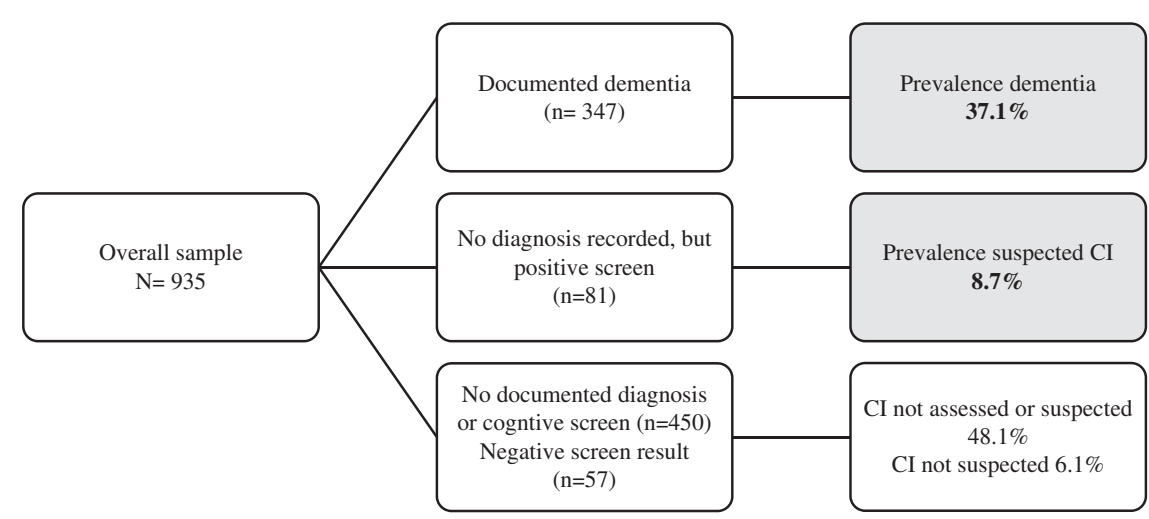

Fig. 1. Algorithm demonstrating selection process of those with dementia of suspected CI. Shaded regions: Proportion of cohort with a diagnosis of dementia or suspected of having cognitive impairment. 
Table 1. Summary and outcomes from cognitive screening test performed $(n=354)$

\begin{tabular}{llcrr}
\hline Cognitive screening tests & Cut-off criteria & $\begin{array}{l}\text { Overall } \\
{[n(\%)]}\end{array}$ & $\begin{array}{l}\text { 'Non-CI' score } \\
{[n(\%)]^{\mathrm{a}}}\end{array}$ & $\begin{array}{l}\text { Below cut-off } \\
{[n(\%)]^{\mathrm{a}}}\end{array}$ \\
\hline MMSE & $\leqslant 23 / 30$ (Folstein et al. 1975) & $131(37.0)$ & $42(32.1)$ & $89(67.9)$ \\
MoCA & $<26$ of 30 (Nasreddine et al. 2005) & $127(35.9)$ & $6(4.7)$ & $121(95.3)$ \\
AMTS & $<8 / 34$ (Jitapunkul et al. 1991) & $46(13.0)$ & $20(43.5)$ & $26(56.5)$ \\
ACE-III & $82 / 100$ (Mioshi et al. 2006; Hsieh et al. 2013) & $41(11.6)$ & $6(14.6)$ & $35(85.4)$ \\
RUDAS & $<22$ of 30 (Storey et al. 2004) & $9(2.5)$ & $3(33.3)$ & $6(66.7)$ \\
Total & & $354(100.0)$ & $77(21.8)^{\mathrm{b}}$ & $277(78.2)^{\mathrm{c}}$ \\
\hline
\end{tabular}

CI, cognitive impairment; MMSE, Mini Mental State Examination; MoCA, Montreal Cognitive Assessment; AMTS, Abbreviated Mental Test Score; ACE-III, Addenbrooke's Cognitive Examination Version III; RUDAS, Rowland Universal Dementia Assessment Scale.

${ }^{\text {a }}$ Expressed as a \% of total number of subjects screened by each specific tool.

${ }^{b}$ Of the 77 clients who obtained a score indicating 'Non-CI', 20/77 had a documented diagnosis of dementia and were included in the prevalence estimates for diagnosed dementia.

${ }^{c}$ Of the 277 clients below the cut-off, 196 had an existing documented dementia diagnosis (classed as dementia) and 81 without documented diagnosis (classed as suspected CI).

Cognitive scores, derived from a range of validated cognitive screening assessments, were documented and criteria indicative of impairment were applied appropriate to the specific tool, as shown in Table 1. Global measures of cognitive function, namely the Mini Mental State Examination (Folstein et al. 1975), was most frequently applied to screen for $\mathrm{CI}$, followed by the Montreal Cognitive Assessment (MoCA) (Nasreddine et al. 2005). Other screening tools included the Abbreviated Mental Test Score (Hodkinson 1972), the Addenbrooke's Cognitive Examination Version III (Mioshi et al. 2006; Hsieh et al. 2013), and the Rowland Universal Dementia Assessment Scale (Storey et al. 2004) which were used in a small number of cases.

Statistical analysis was conducted using IBM SPPS Version 23. Characteristics of the cohort were explored overall using descriptive statistics. Characteristics of those with and without suspected CI were compared, using $t$-tests for continuous variables and $\chi^{2}$ tests for categorical variables. A $p$-value is considered statistically significant at an alpha level of $<0.05$.

\section{Results}

\section{Study cohort and characteristics}

This audit identified 935 older people in receipt of state funded HCPs (Table 2). The cohort was predominantly female $(65 \%)$, single/widowed $(73 \%)$, with a mean age of 83.7 years and the most having multiple health conditions (78\%). Over half (55\%) lived alone. The majority were dependent in ADLs to some degree, ranging from few $(3 \%)$ categorised as independent up to one-quarter $(25.4 \%)$ considered as high-maximum dependency. This is in keeping with the assessment for HCP allocation, which is heavily weighted on level of dependency and need for assistance in day-to-day personal care.

\section{Estimated prevalence of dementia and suspected CI}

Overall, 428 (45.8\%) of this sample of HCP recipients had a diagnosis of dementia or were suspected to have CI. The estimated prevalence of dementia was $37.1 \%$ (347/935) with a further $8.7 \%(81 / 935)$ with suspected CI. Fig. 1 outlines how dementia and suspected CI were classified, with the hierarchy of evidence considered 'documented dementia'. The 81 subjects without a documented diagnosis of dementia, but had a positive cognitive screening test score were classed as suspected CI.

In the present sample, 37.9\% (354/935) underwent some form of cognitive screening test; though for the most part there was no documentation of cognitive screening $(62.1 \%)$. The majority of those screened $(78.2 \%, n=277)$ had scores indicating likelihood of CI. The group with positive screening scores compromised 196/277 who had a documented diagnosis of dementia, and a further $81 / 277$ who did not have evidence of a documented diagnosis; the latter were classed as suspected CI. Only 77 of the clients who partook in cognitive screening tests had scores indicative if non-CI. However, 20 of those also had a documented diagnosis of dementia from a health professional, which for this retrospective audit's inclusion criteria was deemed the strongest evidence for dementia and included in the overall prevalence estimates. To note, $48.1 \%(n=450)$ were not screened and did not have a documented diagnosis of dementia or cognitive decline.

\section{Factors associated with dementia and suspected CI}

A comparison between groups with and without dementia and suspected CI is outlined in Table 2. Significantly fewer of those with dementia and suspected CI were self-caring and correspondingly had higher home help usage, by an average of two 
Table 2. Characteristics of service users overall and according to cognitive status ( $\boldsymbol{n}=935)$

\begin{tabular}{|c|c|c|c|c|}
\hline Characteristics & $\begin{array}{l}\text { All HCP users } \\
100 \%(n=935)\end{array}$ & $\begin{array}{l}\text { Dementia or } \\
\text { suspected CI } 45.8 \% \\
(n=428)\end{array}$ & $\begin{array}{l}\text { Non-dementia, } \\
\text { non-suspected CI } \\
54.4 \%(n=507)\end{array}$ & $p$-value $* *$ \\
\hline Female $[n(\%)]$ & $609(65.1)$ & $295(68.9)$ & $314(61.9)$ & 0.054 \\
\hline Age [mean (S.D.)] & $83.7(7.4)$ & $84.2(7.2)$ & $83.4(7.5)$ & 0.078 \\
\hline Living alone $[n(\%)]$ & $515(55.1)$ & $221(51.6)$ & $294(57.9)$ & 0.068 \\
\hline Married $[n(\%)]$ & $252(27.0)$ & $119(27.8)$ & $133(26.2)$ & 0.398 \\
\hline Self-caring $[n(\%)]$ & $373(39.9)$ & $139(32.5)$ & $234(46.2)$ & $<0.001$ \\
\hline Communication difficulties* $[n(\%)]$ & $141(15.1)$ & $111(25.9)$ & $30(5.9)$ & $<0.001$ \\
\hline Weekly home care hours [mean (s.D.)] & $12.1(6.6)$ & $13.0(6.4)$ & $11.3(6.7)$ & $<0.001$ \\
\hline Barthel score [mean (S.D.)] & $12.9(3.9)$ & $12.6(4.0)$ & $13.1(3.7)$ & 0.056 \\
\hline High-maximum dependency $[n(\%)]$ & $229(24.5)$ & $118(27.6)$ & $111(21.9)$ & 0.032 \\
\hline Medication prompt $[n(\%)]$ & $270(28.9)$ & $164(38.3)$ & $106(20.9)$ & 0.063 \\
\hline Multiple conditions $^{\mathrm{a}}[n(\%)]$ & $728(77.9)$ & $348(81.3)$ & $380(74.9)$ & 0.519 \\
\hline Screened for CI $[n(\%)]$ & $354(37.9)$ & $297(69.4)$ & $57(11.2)$ & $<0.001$ \\
\hline
\end{tabular}

$\mathrm{HCP}$, home care package; $\mathrm{CI}$, cognitive impairment.

Values in italic indicate statistically significant findings.

${ }^{\text {a }}$ Multiple conditions, $>1$ medical conditions.

${ }^{*}$ Communication difficulties ranged from difficulty speaking to no effective means of communication.

** $p$-value derived from Student's $t$-tests for continuous variables and $\chi^{2}$ tests for categorical data.

additional hours per week. A markedly increased frequency of communication difficulties was also noted in this group, relative to those without dementia or suspected CI. Dependency, based on mean Barthel score, did not differ significantly between the groups $(p=0.056)$, although the dementia and suspected CI group were more likely to be classified as high or maximum dependency $(27.7 \%$ v. $21.8 \%, p=0.032)$. Other factors including chronological age, living alone or the presence of co-morbidities showed trends, but did not clearly differentiate the groups. Amongst those with dementia or suspected $\mathrm{CI}$, notably over half $(51.6 \%)$ continued to live alone.

\section{Discussion}

The present audit aimed to explore the prevalence of dementia and $\mathrm{CI}$ among older people living at home supported by state funded HCPs in North Dublin. A high prevalence of dementia $(37.1 \%)$ and suspected CI $(8.7 \%)$ was identified based on a large sample of $935 \mathrm{HCP}$ recipients. In Ireland, there are limited data available on the numbers of people with dementia using home care services, as outlined in the comprehensive literature review conducted by Cahill et al. (2012). The authors highlighted the urgent need for the HSE to produce more dementia-specific data to inform practice and service planning within the community care sector (Cahill et al. 2012). These are the first estimates, to the authors' knowledge, of dementia and suspected CI amongst home care users from an Irish health service setting.
An estimated $9.4 \%$ of the general population aged $65+$ in the community are living with dementia (Central Statistics Office, 2011; Pierce et al. 2014) although there are a lack of reliable Irish epidemiological data (Cahill et al. 2012). In a frailer communitydwelling Irish sample $(n=803)$, median age 80 years, a $16.7 \%$ occurrence of $\mathrm{CI}$ was reported $\left(\mathrm{O}^{\prime}\right.$ Caoimh et al. 2014). Our combined estimate at $45.8 \%$ for dementia and suspected CI is considerably higher as is expected, given this sub-population had a defined need for additional support through formal HCPs. In nursing home settings, $37 \%$ of long stay residents are thought to have severe dementia (Department of Health, 2013), with an earlier study suggesting that the prevalence of CI may be as high as $89 \%$ in residential care homes (Cahill et al. 2010). Furthermore, in a recent study of a similarly sized sample $(n=1001)$ of older adults receiving domiciliary care, using comprehensive valid cognitive approaches, dementia was reported in $41.5 \%$, and mild CI in $27.7 \%$ of domiciliary care users in Norway (Helvik et al. 2015), which is comparable with our own findings for dementia (37.1\%). Indeed, differences in definitions and methodologies around CI assessments, including ours, gives rise to great variations in reported prevalence and makes direct comparison between figures difficult.

As anticipated, factors associated with dementia and suspected CI, in the present study included significantly higher dependency rates and weekly home help hours. Other associated factors included communication difficulty and lower rates of self-caring 
status. The relationship observed between CI and increased dependency is consistent with recent findings in a home help cohort (Helvik et al. 2015). Although trends were noted, chronological age, gender, need for medication prompts, marital and living status or co-morbidities did not as clearly differentiate those with and without dementia and suspected CI.

Importantly, this audit provides profile data of state funded HCP users (Table 1) in Ireland. Our sample was characterised as predominantly female, single/ widowed, aged 83.7, with multiple health issues and over half lived alone. Combining this with a $45.8 \%$ prevalence of likely dementia and CI, highlights HCP users as a vulnerable group. These findings are broadly in agreement with data on older people receiving domiciliary care in Norway, who were on average 83.4 years, predominantly female $(68 \%)$, single $(73 \%)$ with fair-very poor self-reported health (85\%) and displayed high degrees of (well defined) dementia and CI (Helvik et al. 2015).

While the sample size is a key strength of this audit, several limitations need to be addressed. As recording of cognitive information was not mandatory for HCP assessment, determination of cognitive health was derived from available records; those with no information documented on cognition were classed as ' $\mathrm{CI}$ not suspected'; thus, likely underestimating true prevalence. Cognitive screening scores were documented for only a subset of clients. Where cognitive screening tools were applied, their heterogeneity, inherent differences in sensitivity, specificity and inter-rater reliability poses further challenges for interpretation. Furthermore, various cut-offs can be applied when investigating cognitive status by means of a cognitive screening tool. When the lower cut-off was applied for the MoCA assessment ( $<22$ points) the prevalence of suspected CI dropped from $8.6 \%$ to $5 \%$. Our findings represent a crude estimate of suspected CI. Other associated socio-demographic factors, such as educational attainment, would improve the accuracy of identifying $\mathrm{CI}$ in a community-setting. Cognitive screening tools, such as the MoCA, which offer education-adjusted scoring, could be suggested as a standard assessment tool in the community setting. Future research efforts should place a focus on ensuring reliable cognitive assessments and diagnosis by healthcare professionals, as well as improved documentation of such information in CSAR forms.

Indeed, the audit highlights a lack of consistency and documentation around CI in HCP clients in the community. Whilst the National Dementia Strategy does not recommend population-based screening for dementia (Department of Health 2014), there is increasing consensus that early recognition and diagnosis of dementia is likely to increase the chances of successful interventions and supports (Overshott and Burns, 2005; Cahill et al. 2012; Morley et al. 2015) and optimise future care planning and decision making (Overshott and Burns, 2005; Cahill et al. 2012). A high prevalence of dementia and suspected CI among HCP users, emphasises a need for multi-modal approaches, for example, dementia-specific training for home care staff in the community (All-Party Parliamentary Group, 2009; Cahill et al. 2012), more community-based health professionals, dementia-specific HCPs (Department of Health, 2014) and assistive technology in the home (Cahill et al. 2007). Consistent with this, recent commentary in the Lancet, highlighted that dementia care needs to be individualised, compassionate, and integrated into community efforts, with 'a dedicated, educated, adequately financed, and well respected homecare workforce' (Lancet, 2016).

In conclusion, this audit suggests a high prevalence of dementia $(37.1 \%)$ in older adults receiving HCPs. Notwithstanding the study limitations, the findings represent the first estimates, to the authors' knowledge, of dementia in home care from an Irish health service setting. The data provide important considerations for service planning and identify a need and opportunity for dementia specific home care and interventions to support positive 'ageing in place', in line with the Programme for Government (Department of Health, 2012).

\section{Financial Support}

Researchers (S.K., I.O.B., K.S.) were funded by North Dublin Home Care Ltd., a member of the Dublin Home Care Partners Consortium and a not-for-profit organisation providing home care to $\mathrm{CHO}$ Area 9 which includes Dublin North, Dublin North Central, and Dublin North West. All results, interpretation of data, and conclusions reported in this paper are independent from funding sources. The funders did not contribute to the design and conduct of the study.

\section{Conflicts of Interest}

The authors have no conflicts of interest to disclose.

\section{Ethical Standards}

The authors assert that all procedures contributing to this work comply with the ethical standards of the relevant national and institutional committee on human experimentation with the Helsinki Declaration of 1975, as revised in 2008. The study was approved by the HSE, Community Health Organisation Area 9, Dublin and ethical approval was granted by the Research Ethics Committee in Dublin City University, Dublin (DCUREC/2015/236). Written consent was obtained from all study participants before conducting each CSAR assessment. 


\section{References}

All-Party Parliamentary Group (2009). Prepared to Care: Challenging the Dementia Skills Gap. All-Party Parliamentary Group: London.

Cahill S, Diaz-Ponce AM, Coen RF, Walsh C (2010). The underdetection of cognitive impairment in nursing homes in the Dublin area. The need for on-going cognitive assessment. Age and Ageing 39, 128-131.

Cahill SBE, Faulkner JP, Hagen I (2007). “It gives me a sense of independence" Findings from Ireland on the use and usefulness of assistive technology for people with dementia. Technology and Disability 19, 133-142.

Cahill S, O'Shea E, Pierce M (2012). Creating Excellence in Dementia Care: A Research Review for Ireland's National Dementia Strategy. TCD-NUIG: Ireland.

Collin C, Wade DT, Davies S, Horne V (1988). The Barthel ADL Index: a reliability study. International Disability Studies 10, 61-63.

Central Statistics Office (2011). StatBank (http://www.cso.ie/ $\mathrm{px} /$ pxeirestat/Statire/SelectVarVal/Define.asp?Maintable= CDD16\&Planguage=0). Accessed 14 December 2016.

Department of Health (2012). Future Health: A Strategic Framework for Reform of the Health Service 2012-2015. Department of Health: Dublin.

Department of Health (2013). Long Stay Activity Statistics. Department of Health: Dublin.

Department of Health (2014). The Irish National Dementia Strategy. Department of Health: Dublin.

Folstein MF, Folstein SE, McHugh PR (1975). "Mini-mental state". A practical method for grading the cognitive state of patients for the clinician. Journal of Psychiatric Research 12, 189-198.

Health Services Executive (2009). Common Summary Assessment Report: Guidance Document. Health Services Executive: Ireland.

Helvik AS, Hogseth LD, Bergh S, Saltyte-Benth J, Kirkevold O, Selbaek G (2015). A 36-month follow-up of decline in activities of daily living in individuals receiving domiciliary care. BMC Geriatrics 15, 47.

Hodkinson HM (1972). Evaluation of a mental test score for assessment of mental impairment in the elderly. Age and Ageing 1, 233-238.

Hsieh S, Schubert S, Hoon C, Mioshi E, Hodges JR (2013). Validation of the Addenbrooke's cognitive examination III in frontotemporal dementia and Alzheimer's disease. Dementia and Geriatric Cognitive Disorders 36, 242-250.

Jitapunkul S, Pillay I, Ebrahim S (1991). The abbreviated mental test: its use and validity. Age and Ageing 20, 332-336.

Lancet (2016). The unacceptable reality of care for people living with dementia. The Lancet 388, 2566.

Mioshi E, Dawson K, Mitchell J, Arnold R, Hodges JR (2006). The Addenbrooke's Cognitive Examination Revised (ACE-R): a brief cognitive test battery for dementia screening. International Journal of Geriatric Psychiatry 21, 1078-1085.

Morley JE, Morris JC, Berg-Weger M, Borson S, Carpenter BD, Del Campo N, Dubois B., Fargo K, Fitten LJ, Flaherty JH, Ganguli M, Grossberg GT, Malmstrom TK, Petersen RD, Rodriguez C, Saykin AJ, Scheltens P, Tangalos EG, Verghese J, Wilcock G, Winblad B, Woo J, Vellas B (2015). Brain health: the importance of recognizing cognitive impairment: an IAGG consensus conference. Journal of the American Medical Directors Association 16, 731-739.

Murphy CM, Whelan BJ, Normand C (2015). Formal homecare utilisation by older adults in Ireland: evidence from the Irish Longitudinal Study on Ageing (TILDA). Health and Social Care in the Community 23, 408-418.

Nasreddine ZS, Phillips NA, Bedirian V, Charbonneau S, Whitehead V, Collin I, Cummings JL, Chertkow H (2005). The Montreal Cognitive Assessment, MoCA: a brief screening tool for mild cognitive impairment. Journal of the American Geriatric Society 53, 695-699.

O'Caoimh R, Gao Y, Svendrovski A, Healy E, O'Connell E, O'Keeffe G, Cronin U, O'Herlihy E, Cornally N, Molloy WD (2014). Screening for markers of frailty and perceived risk of adverse outcomes using the Risk Instrument for Screening in the Community (RISC). BMC Geriatrics 14, 104.

Overshott R, Burns A (2005). Treatment of dementia. Journal of Neurology, Neurosurgery \& Psychiatry 76, 53-59.

Pierce M, Cahill S, O'Shea E (2014). Prevalence and Projections of Dementia in Ireland, 2011-2046. Genio: Mullingar.

Storey JE, Rowland JT, Basic D, Conforti DA, Dickson HG (2004). The Rowland Universal Dementia Assessment Scale (RUDAS): a multicultural cognitive assessment scale. International Psychogeriatrics 16, 13-31. 